\title{
Clinical characteristics and outcomes in elderly patients undergoing transsphenoidal surgery for nonfunctioning pituitary adenoma
}

\author{
Matheus P. Pereira, BS, ${ }^{1}$ Taemin Oh, MD, ${ }^{2}$ Rushikesh S. Joshi, BS, ${ }^{2}$ Alexander F. Haddad, BS, ${ }^{1}$ \\ Kaitlyn M. Pereira, BS, ${ }^{3}$ Robert C. Osorio, BS, ${ }^{1}$ Kevin C. Donohue, BS, ${ }^{1}$ Zain Peeran, BS, ${ }^{2}$ \\ Sweta Sudhir, BS, ${ }^{2}$ Saket Jain, PhD, ${ }^{2}$ Angad Beniwal, BS, ${ }^{2}$ José Gurrola II, MD, ${ }^{4}$ \\ Ivan H. El-Sayed, MD, ${ }^{4}$ Lewis S. Blevins Jr., MD, ${ }^{2}$ Philip V. Theodosopoulos, MD, ${ }^{2}$ \\ Sandeep Kunwar, MD, ${ }^{2}$ and Manish K. Aghi, MD, PhD²
}

${ }^{1}$ School of Medicine and Departments of ${ }^{2}$ Neurological Surgery and ${ }^{4}$ Otolaryngology-Head and Neck Surgery, University of California, San Francisco, California; and ${ }^{3}$ University of South Florida Morsani College of Medicine, Tampa, Florida

OBJECTIVE Life expectancy has increased over the past century, causing a shift in the demographic distribution toward older age groups. Elderly patients comprise up to $14 \%$ of all patients with pituitary tumors, with most lesions being nonfunctioning pituitary adenomas (NFPAs). Here, the authors evaluated demographics, outcomes, and postoperative complications between nonelderly adult and elderly NFPA patients.

METHODS A retrospective review of 908 patients undergoing transsphenoidal surgery (TSS) for NFPA at a single institution from 2007 to 2019 was conducted. Clinical and surgical outcomes and postoperative complications were compared between nonelderly adult (age $\geq 18$ and $\leq 65$ years) and elderly patients (age $>65$ years).

RESULTS There were 614 and 294 patients in the nonelderly and elderly groups, respectively. Both groups were similar in sex ( $57.3 \%$ vs $60.5 \%$ males; $p=0.4$ ), tumor size ( 2.56 vs $2.46 \mathrm{~cm} ; p=0.2)$, and cavernous sinus invasion ( $35.8 \%$ vs $33.7 \% ; p=0.6)$. Regarding postoperative outcomes, length of stay (1 vs 2 days; $p=0.5)$, extent of resection $(59.8 \%$ vs $64.8 \%$ gross-total resection; $p=0.2$ ), CSF leak requiring surgical revision (4.3\% vs $1.4 \% ; p=0.06)$, 30-day readmission ( $8.1 \%$ vs $7.3 \% ; p=0.7$ ), infection ( $3.1 \%$ vs $2.0 \% ; p=0.5)$, and new hypopituitarism $(13.9 \%$ vs $12.0 \% ; p=0.3)$ were similar between both groups. Elderly patients were less likely to receive adjuvant radiation $(8.7 \%$ vs $16.3 \% ; p=0.009)$, undergo future reoperation ( $3.8 \%$ vs $9.5 \% ; p=0.003)$, and experience postoperative diabetes insipidus (DI) $(3.7 \%$ vs $9.4 \% ; p=$ $0.002)$, and more likely to have postoperative hyponatremia ( $26.7 \%$ vs $16.4 \% ; p<0.001)$ and new cranial nerve deficit $(1.9 \%$ vs $0.0 \% ; p=0.01)$. Subanalysis of elderly patients showed that patients with higher Charlson Comorbidity Index scores had comparable outcomes other than higher DI rates $(8.1 \%$ vs $0.0 \% ; p=0.006)$. Elderly patients' postoperative sodium peaked and troughed on postoperative day 3 (POD3) (mean $138.7 \mathrm{mEq} / \mathrm{L}$ ) and POD9 (mean $130.8 \mathrm{mEq} / \mathrm{L}$ ), respectively, compared with nonelderly patients (peak POD2: mean $139.9 \mathrm{mEq} / \mathrm{L}$; trough POD8: mean $131.3 \mathrm{mEq} / \mathrm{L}$ ).

CONCLUSIONS The authors' analysis revealed that TSS for NFPA in elderly patients is safe with low complication rates. In this cohort, more elderly patients experienced postoperative hyponatremia, while more nonelderly patients experienced postoperative DI. These findings, combined with the observation of higher DI in patients with more comorbidities and elderly patients experiencing later peaks and troughs in serum sodium, suggest age-related differences in sodium regulation after NFPA resection. The authors hope that their results will help guide discussions with elderly patients regarding risks and outcomes of TSS.

https://thejns.org/doi/abs/10.3171/2020.7.FOCUS20524

KEYWORDS elderly; geriatric; nonfunctioning pituitary adenoma; transsphenoidal surgery

ABBREVIATIONS CCI = Charlson Comorbidity Index; $\mathrm{CN}=$ cranial nerve; $\mathrm{CS}$ = cavernous sinus; $\mathrm{DI}=$ diabetes insipidus; $\mathrm{EBL}=$ estimated blood loss; $\mathrm{EOR}=$ extent of resection; ETSS = endoscopic TSS; GTR = gross-total resection; LOS = length of stay; MTSS = microscopic TSS; NFPA = nonfunctioning PA; PA = pituitary adenoma; POD = postoperative day; SIADH = syndrome of inappropriate antidiuretic hormone secretion; STR = subtotal resection; TSS = transsphenoidal surgery.

SUBMITTED June 1, 2020. ACCEPTED July 20, 2020.

INCLUDE WHEN CITING DOI: 10.3171/2020.7.FOCUS20524. 
$\mathrm{P}$ ITUITARY adenomas (PAs) are common benign intracranial neoplasms, with an estimated prevalence of $16.7 \%$ in the general population..$^{1,2}$ Nonfunctioning PAs (NFPAs) are a type of PA without clinical evidence of excess hormone secretion, and, due to their silent nature, patients present with symptoms related to mass effect, such as visual disturbances. ${ }^{3}$ Current treatment for symptomatic or nonfunctioning PAs that are large enough to warrant treatment consists of resection, with radiation therapy reserved for residual or recurrent tumors small enough to not need surgery. 4,5

Life expectancy has increased over the past century, causing a shift in the demographic distribution in developed countries toward older age groups. ${ }^{6}$ Studies have suggested that this segment of the population is the fastestgrowing age group in industrialized countries, and, if the current pace of annual growth in life expectancy continues, the majority of people born since 2000 will live past the age of 100 years. ${ }^{7}$ Projections indicate that the number of individuals aged 65 and older will more than double by $2050 .{ }^{8}$ Furthermore, current projections show that the number of adults older than 65 years will more than double the number of children younger than 5 years and will account for a larger section of the worldwide population than adolescents and youth aged 15-24 years by $2050 .{ }^{8}$

Elderly patients account for up to $14 \%$ of all patients diagnosed with pituitary tumors, with most lesions being NFPAs. ${ }^{9-14}$ Although the incidence of PAs is higher in younger patients, the aging population renders investigation of the safety of surgical intervention for adenomas and other brain neoplasms crucial. Previous studies have explored patient outcomes after transsphenoidal surgery (TSS) for PA in the elderly and have reported the safety of this approach in select populations, albeit with small sample sizes and mixed results regarding risks and complication rates..$^{10,11,14-31}$

Here, we provide the largest single-center experience examining patient and surgical outcomes in elderly patients undergoing TSS for NFPAs. We aim to compare nonelderly and elderly populations with regard to demographics, outcomes, and postoperative complications.

\section{Methods}

\section{Patient and Data Collection}

Using ICD diagnostic codes, we identified and retrospectively reviewed 908 consecutive adult patients who underwent microscopic or endoscopic TSS (MTSS and ETSS, respectively) for NFPA at the University of California, San Francisco, from May 1, 2007, to October 15, 2019, following approval from our institutional review board. We compared demographic and surgical outcomes in elderly patients ( $>65$ years) with those of nonelderly patients ( $\geq 18$ and $\leq 65$ years). Demographic and surgical variables collected from patient charts included age, sex, symptoms at presentation, length of stay (LOS), surgical approach (MTSS or ETSS), average follow-up time, history of prior NFPA resection, extent of resection (EOR), future reoperation, drain placement (lumbar or extraventricular), intraoperative CSF encounter, time of operation, estimated blood loss (EBL), final tumor pathology, and preoperative hypopituitarism. Postoperative complications recorded included CSF leak requiring surgical repair, new cranial nerve $(\mathrm{CN})$ deficit, 30-day hospital readmission, postoperative infection, postoperative hyponatremia, development of diabetes insipidus (DI), and new postoperative hypopituitarism. Postoperative hyponatremia was defined as serum sodium levels $<135 \mathrm{mEq} / \mathrm{L}$ within 14 days of the operation. DI was defined as present in patients with serum sodium $>145 \mathrm{mEq} / \mathrm{L}$ within 14 days of operation and urine output $>300 \mathrm{~mL} / \mathrm{hr}$ and/or who required use of desmopressin. In patients with DI, we defined transient DI in patients with full resolution of DI symptoms at the time of follow-up, and permanent DI in those with persistence of symptoms requiring daily desmopressin treatment. We also noted if patients received adjuvant radiosurgery or radiation therapy. In elderly patients, we recorded patient medical comorbidities according to the Charlson Comorbidity Index (CCI). ${ }^{32}$ Finally, we compared clinical and surgical outcomes in two subgroups of elderly patients (age $>65$ and $\leq 75$ years vs $>75$ years) and patients with CCI score $<6$ versus $\geq 6$.

\section{Neuroimaging Assessment}

Tumor measurements were noted by radiologists on preoperative MRI scans when present in the medical records. A single research associate performed all tumor measurements in patients when radiographic measurements were unavailable. Tumor diameter was defined as the maximum diameter in one of the 3 radiographic dimensions (anteroposterior, mediolateral, and superoinferior). EOR was determined by radiologists on postoperative MRI scans, and it was defined as gross-total resection (GTR) and subtotal resection (STR). The presence of cavernous sinus (CS) invasion was also noted by radiologists on preoperative MRI scans.

\section{Statistical Analysis}

Categorical variables were compared using the Pearson chi-square test and Fisher's exact test. Continuous variables were analyzed using a two-tailed t-test, and nonparametric variables were analyzed using the Mann-Whitney U-test; $p<0.05$ was considered statistically significant. Statistical analysis was completed using IBM SPSS Statistics software (version 26.0, IBM Corp.). Plots were generated using the Matplotlib library in Python version 2.7.10.

\section{Results}

\section{Patient Cohort and Tumor Characteristics}

We identified 908 patients who underwent TSS for NFPAs at our institution from May 1, 2007, to October 15,2019 . In our cohort, 614 patients $(67.6 \%)$ were nonelderly and 294 patients (32.4\%) were elderly (Table 1). In the nonelderly group, 352 patients $(57.3 \%)$ were male and $262(42.7 \%)$ were female, while in the elderly group, 178 patients $(60.5 \%)$ were male and $116(39.5 \%)$ were female $(\mathrm{p}=0.4)$. The median age of the nonelderly group was 52 years (range 18-65 years), while the median age in the elderly group was 73 years (range 66-93 years). To characterize temporal trends, we created a year-by-year breakdown in elderly versus nonelderly patients undergo- 
TABLE 1. Patient demographic information and surgical outcomes, stratified by age group

\begin{tabular}{|c|c|c|c|}
\hline Variable & Age $\leq 65$ Yrs & Age $>65$ Yrs & $\mathrm{p}$ Value \\
\hline Total patients, n (\%) & $614(67.6)$ & $294(32.4)$ & \\
\hline Sex, $n(\%)$ & & & 0.388 \\
\hline $\mathrm{M}$ & $352(57.3)$ & $178(60.5)$ & \\
\hline $\mathrm{F}$ & $262(42.7)$ & $116(39.5)$ & \\
\hline Age in yrs, median [IQR] & $52.00[44.00-59.00]$ & $73.00[69.00-79.00]$ & \\
\hline LOS in days, median [IQR] & $1.00[1.00-3.00]$ & $2.00[1.00-3.00]$ & 0.492 \\
\hline Follow-up time in mos, median [IQR] & $22.00[10.75-42.00]$ & $19.00[10.00-44.00]$ & 0.541 \\
\hline Tumor diameter in $\mathrm{cm}$, mean \pm SD & $2.56 \pm 1.15$ & $2.46 \pm 0.94$ & 0.208 \\
\hline Approach, $\mathrm{n}(\%)$ & & & 0.716 \\
\hline MTSS & $495(80.6)$ & $240(81.6)$ & \\
\hline ETSS & $119(19.4)$ & $54(18.4)$ & \\
\hline Prior pituitary surgery, n (\%) & & & 0.007 \\
\hline Yes & $117(19.1)$ & $35(11.9)$ & \\
\hline No & $497(80.9)$ & $259(88.1)$ & \\
\hline CS invasion, $\mathrm{n}(\%)$ & & & 0.550 \\
\hline Yes & $215(35.8)$ & $98(33.7)$ & \\
\hline No & $386(64.2)$ & $193(66.3)$ & \\
\hline EOR, n (\%) & & & 0.158 \\
\hline GTR & $352(59.8)$ & $182(64.8)$ & \\
\hline STR & $237(40.2)$ & $99(35.2)$ & \\
\hline Drain, $n(\%)$ & & & 0.417 \\
\hline EVD & $0(0.0)$ & $1(0.3)$ & \\
\hline Lumbar & $139(22.6)$ & $64(21.8)$ & \\
\hline None & $475(77.4)$ & $229(77.9)$ & \\
\hline Intraop CSF encounter, n (\%) & & & 0.999 \\
\hline Yes & $123(20.0)$ & $58(19.7)$ & \\
\hline No & $491(80.0)$ & $236(80.3)$ & \\
\hline Duration of op in mins, mean \pm SD & $138.3 \pm 54.2$ & $132.3 \pm 53.0$ & 0.195 \\
\hline $\mathrm{EBL}$ in $\mathrm{mL}$, mean $\pm \mathrm{SD}$ & $60.2 \pm 58.6$ & $72.3 \pm 145.5$ & 0.147 \\
\hline Final pathology, n (\%) & & & 0.308 \\
\hline Gonadotroph adenoma & $267(65.3)$ & $144(69.6)$ & \\
\hline Null-cell adenoma & $93(22.7)$ & $43(20.8)$ & \\
\hline Silent corticotroph adenoma & $39(9.5)$ & $19(9.2)$ & \\
\hline Silent PIT1 (GH/prolactin/TSH lineage) adenoma & $10(2.4)$ & $1(0.5)$ & \\
\hline Adjuvant radiation, n (\%) & & & 0.009 \\
\hline Yes & $67(16.3)$ & $18(8.7)$ & \\
\hline Radiosurgery & $45(11.0)$ & $7(3.4)$ & 0.001 \\
\hline Radiation therapy & $22(5.4)$ & $11(5.3)$ & 0.999 \\
\hline No & $343(83.7)$ & $189(91.3)$ & \\
\hline Future reop, $\mathrm{n}(\%)$ & & & 0.003 \\
\hline Yes & $57(9.5)$ & $11(3.8)$ & \\
\hline No & $543(90.5)$ & $279(96.2)$ & \\
\hline Postop visual improvement, $\mathrm{n}(\%)$ & & & 0.528 \\
\hline Yes & $153(82.7)$ & $70(79.5)$ & \\
\hline No & $32(17.3)$ & $18(20.5)$ & \\
\hline
\end{tabular}

EVD = external ventricular drain; $\mathrm{GH}$ = growth hormone; TSH = thyroid-stimulating hormone.

Boldface type indicates statistical significance. Percentages are based on the number of patients with available information for the given variable. 

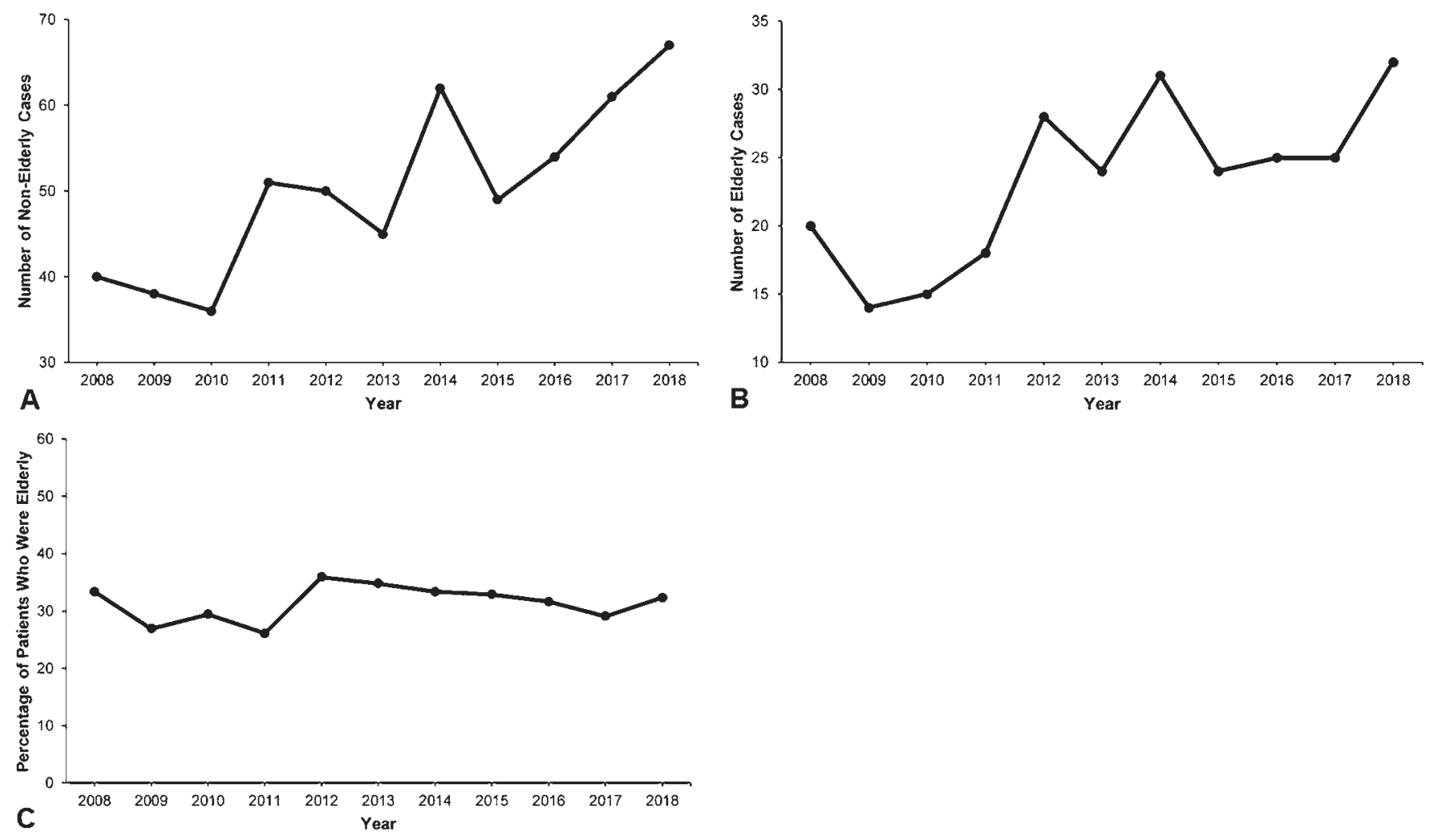

FIG. 1. Trends in nonelderly patients (A), elderly patients (B), and the proportion of elderly patients in the overall number of cases (C) for patients undergoing TSS for NFPA resection from 2008 to 2018.

ing TSS for NFPAs at our center from 2008 to 2018. Our results showed a steady increase in the total number of patients undergoing NFPA resection over the decade, with a parallel increase in the number of elderly and nonelderly patients over time without a change in the proportion of older patients (Fig. 1).

Older patients were more likely to be asymptomatic at the time of diagnosis $(13.1 \%$ vs $5.1 \%$; $p=0.001)$, with the mean tumor size of asymptomatic tumors undergoing surgery being $2.20 \mathrm{~cm}$ in nonelderly versus $2.07 \mathrm{~cm}$ in elderly patients $(\mathrm{p}=0.6)$ (Table 2). Among asymptomatic patients, the most common indications for MRI scans included trauma $(15.0 \%)$ and incidental abnormalities in laboratory values $(15.0 \%)$ in younger patients, and mechanical falls (23.1\%), metastasis screening or workup for other masses (19.2\%), workup for unrelated neurological complaints (e.g., previous stroke, history of seizures, and tremors; 19.2\%), and memory impairment $(7.7 \%)$ in elderly patients. When stratifying asymptomatic patients undergoing surgery by decade of life, a higher percentage of surgical patients having no symptoms at presentation were in the 61- to 70-yearold (32.6\%) and 71- to 80-year-old (37.0\%) age groups than in earlier and later decades (Table 3). Among symptoms that symptomatic patients reported, younger patients were more likely to present with headache $(44.6 \%$ vs $33.7 \%$; $p$ $=0.01)$, amenorrhea or irregular menses $(10.8 \%$ vs $0.0 \%$; $\mathrm{p}<0.001$ ), galactorrhea (4.6\% vs $0.0 \% ; \mathrm{p}=0.001)$, and decreased libido or sexual dysfunction $(10.8 \%$ vs $5.5 \%$; $\mathrm{p}=$ $0.047)$. There was no difference in the prevalence of visual deficits $(54.6 \%$ vs $50.8 \%$; $\mathrm{p}=0.4)$, fatigue or decreased energy (29.0\% vs $26.6 \%$; $\mathrm{p}=0.6)$, weight changes $(12.8 \%$ vs $9.0 \% ; \mathrm{p}=0.2)$, dizziness $(12.8 \%$ vs $14.6 \% ; \mathrm{p}=0.6)$, or preoperative hypopituitarism ( $42.8 \%$ vs $35.6 \% ; \mathrm{p}=0.08)$ between the groups. Younger patients were more likely to have had prior surgery for NFPAs $(19.1 \%$ vs $11.9 \%$; $\mathrm{p}=$ $0.007)$. The mean tumor diameter was not significantly

TABLE 2. Presenting symptoms in patients with NFPA, stratified by age

\begin{tabular}{|c|c|c|c|}
\hline \multirow[b]{2}{*}{ Presenting Symptom } & \multicolumn{2}{|c|}{ No. of Patients (\%) } & \multirow[b]{2}{*}{ p Value } \\
\hline & $\begin{array}{c}\text { Age } \\
\leq 65 \text { Yrs }\end{array}$ & $\begin{array}{c}\text { Age } \\
>65 \text { Yrs }\end{array}$ & \\
\hline Visual deficit & $213(54.6)$ & $101(50.8)$ & 0.384 \\
\hline Headache & $174(44.6)$ & $67(33.7)$ & 0.013 \\
\hline Amenorrhea/irregular menses & $42(10.8)$ & $0(0.0)$ & $<0.001$ \\
\hline Galactorrhea & $18(4.6)$ & $0(0.0)$ & 0.001 \\
\hline Libido decrease/sexual dysfunction & $42(10.8)$ & $11(5.5)$ & 0.047 \\
\hline Fatigue/decreased energy & $113(29.0)$ & $53(26.6)$ & 0.563 \\
\hline Weight change & $50(12.8)$ & $18(9.0)$ & 0.220 \\
\hline Dizziness & $50(12.8)$ & $29(14.6)$ & 0.609 \\
\hline Asymptomatic & $20(5.1)$ & $26(13.1)$ & 0.001 \\
\hline Preop hypopituitarism & $172(43.5)$ & $74(36.1)$ & 0.081 \\
\hline
\end{tabular}

Boldface type indicates statistical significance. Percentages are based on the number of patients with available information for the given variable. 
TABLE 3. Age distribution of asymptomatic patients undergoing surgery for NFPA

\begin{tabular}{cc}
\hline Decade of Life (yrs) & No. of Patients (\%) \\
\hline $11-20$ & $0(0.0)$ \\
\hline $21-30$ & $0(0.0)$ \\
\hline $31-40$ & $3(6.5)$ \\
\hline $41-50$ & $2(4.3)$ \\
\hline $51-60$ & $6(13.0)$ \\
\hline $61-70$ & $15(32.6)$ \\
\hline $71-80$ & $17(37.0)$ \\
\hline $81-90$ & $3(6.5)$ \\
\hline $91-100$ & $0(0.0)$ \\
\hline
\end{tabular}

different between the groups ( 2.56 vs $2.46 \mathrm{~cm}$; $\mathrm{p}=0.2$ ). Rates of CS invasion were also similar between the nonelderly and elderly populations (35.8\% vs $33.7 \%$; $\mathrm{p}=0.6)$. There was no difference in regard to final pathology between the groups, with tumors consisting of gonadotroph adenomas $(65.3 \%$ vs $69.6 \%)$, null-cell adenomas $(22.7 \%$ vs $20.8 \%)$, silent corticotroph adenomas $(9.5 \%$ vs $9.2 \%$ ), and silent PIT1 (growth hormone/prolactin/thyroid-stimulating lineage) adenomas (2.4\% vs $0.5 \% ; \mathrm{p}=0.3)$.

\section{Surgical Variables}

The median LOS for nonelderly patients was 1 day, while the median LOS for elderly patients was 2 days (p $=0.5)$ (Table 1). Regarding surgical approach, 495 patients (80.6\%) underwent MTSS and 119 patients (19.4\%) underwent ETSS in the nonelderly group, whereas 240 patients $(81.6 \%)$ underwent MTSS and 54 patients (18.4\%) underwent ETSS in the elderly group ( $\mathrm{p}=0.7)$. EOR was similar between the groups, with 352 nonelderly patients (59.8\%) and 182 elderly patients (64.8\%) having undergone GTR ( $p$ $=0.2$ ). The patient populations also did not differ in number of cases requiring lumbar drains $(22.6 \%$ vs $21.8 \%)$ or external ventricular drains $(0.0 \%$ vs $0.3 \%$; $=0.4)$. Rates of intraoperative CSF encounters were similar between younger and older patients (20.0\% vs $19.7 \%$; $\mathrm{p}=0.999)$. Elderly patients had a similar duration of the operation (mean 132.3 vs 138.3 minutes; $p=0.2$ ) and EBL (mean 72.3 vs $60.2 \mathrm{~mL} ; \mathrm{p}=0.2$ ) compared with nonelderly patients.

\section{Long-Term Outcomes}

Nonelderly adults were more likely to undergo postoperative adjuvant radiation therapy than elderly patients $(16.3 \%$ vs $8.7 \%$; $p=0.009)$ within a median of 5.5 months (IQR 3-12 months) after surgery. Among those patients, nonelderly adults were more likely to undergo radiosurgery $(11.0 \%$ vs $3.4 \%$; $\mathrm{p}=0.001)$, while both groups had similar rates of external-beam radiation therapy $(5.4 \%$ vs $5.3 \% ; p=0.999)$. In terms of surveillance of recurrence, the median follow-up time was 52 months for nonelderly patients and 73 months for elderly patients $(p=0.5)$ (Table 1). There was no significant difference in postoperative CSF leaks requiring surgical repair between our two cohorts (Table 4). Nonelderly and elderly patients had
TABLE 4. Postoperative complications, stratified by age

\begin{tabular}{|c|c|c|c|}
\hline \multirow[b]{2}{*}{ Complication } & \multicolumn{2}{|c|}{ No. of Patients (\%) } & \multirow[b]{2}{*}{ p Value } \\
\hline & Age $\leq 65$ Yrs & Age $>65 \mathrm{Yrs}$ & \\
\hline Postop CSF leak & & & 0.063 \\
\hline Yes & $19(4.3)$ & $3(1.4)$ & \\
\hline No & $424(95.7)$ & $214(98.6)$ & \\
\hline 30-day readmission & & & 0.736 \\
\hline Yes & $35(8.1)$ & $17(7.3)$ & \\
\hline No & $399(91.9)$ & $215(92.7)$ & \\
\hline Infection & & & 0.516 \\
\hline Meningitis & $4(0.7)$ & $3(1.0)$ & \\
\hline Pneumonia & $1(0.2)$ & $1(0.3)$ & \\
\hline Sinusitis & $12(2.0)$ & $2(0.7)$ & \\
\hline Sepsis & $1(0.2)$ & $0(0.0)$ & \\
\hline Fat graft incision infection & $1(0.2)$ & $0(0.0)$ & \\
\hline None & $595(96.9)$ & $288(98.0)$ & \\
\hline Postop hyponatremia & & & $<0.001$ \\
\hline Yes & $100(16.4)$ & $78(26.7)$ & \\
\hline No & $511(83.6)$ & $214(73.3)$ & \\
\hline Postop CN deficit & & & 0.012 \\
\hline Yes & $0(0.0)$ & $4(1.9)$ & \\
\hline No & $410(100.0)$ & $203(98.1)$ & \\
\hline $\mathrm{DI}$ & & & 0.002 \\
\hline Yes & $58(9.4)$ & $11(3.7)$ & \\
\hline Transient & $33(5.4)$ & $6(2.0)$ & \\
\hline Permanent & $25(4.1)$ & $5(1.7)$ & \\
\hline No & $556(90.6)$ & $283(96.3)$ & \\
\hline Postop hypopituitarism & & & 0.340 \\
\hline Yes & $50(13.9)$ & $23(12.0)$ & \\
\hline Central hypothyroidism & $14(3.9)$ & $7(3.7)$ & 0.999 \\
\hline Male hypogonadism & $9(2.5)$ & $2(1.0)$ & 0.345 \\
\hline Female hypogonadism & $4(1.1)$ & $0(0.0)$ & 0.304 \\
\hline Low GH/IGF-1 & $34(9.5)$ & $8(4.2)$ & 0.028 \\
\hline Central hypoadrenalism & $23(6.4)$ & $16(8.4)$ & 0.389 \\
\hline No & $309(86.1)$ & $168(88.0)$ & \\
\hline
\end{tabular}

IGF-1 = insulin-like growth factor-1.

Boldface type indicates statistical significance. Percentages are based on the number of patients with available information for the given variable.

similar rates of 30 -day readmission $(8.1 \%$ vs $7.3 \% ; p=0.7)$ and postoperative infection, including meningitis $(0.7 \%$ vs $1.0 \%)$, pneumonia $(0.2 \%$ vs $0.3 \%)$, sinusitis $(2.0 \%$ vs $0.7 \%)$, sepsis $(0.2 \%$ vs $0.0 \%)$, and fat graft incision infection $(0.2 \%$ vs $0.0 \%)(p=0.5)$.

The elderly group had higher likelihood of developing postoperative hyponatremia $(26.7 \%$ vs $16.4 \%$; $p<0.001)$. To investigate the trend in postoperative sodium values, we created a plot with postoperative sodium values as a function of postoperative day (POD). Older patients had a postoperative sodium peak on POD3 (mean $138.7 \mathrm{mEq} / \mathrm{L}$ ) and trough on POD9 (mean $130.8 \mathrm{mEq} / \mathrm{L}$ ), compared with younger patients, whose peak was on POD2 (mean 139.9 $\mathrm{mEq} / \mathrm{L}$ ) and trough on POD8 (131.3 mEq/L) (Fig. 2). 

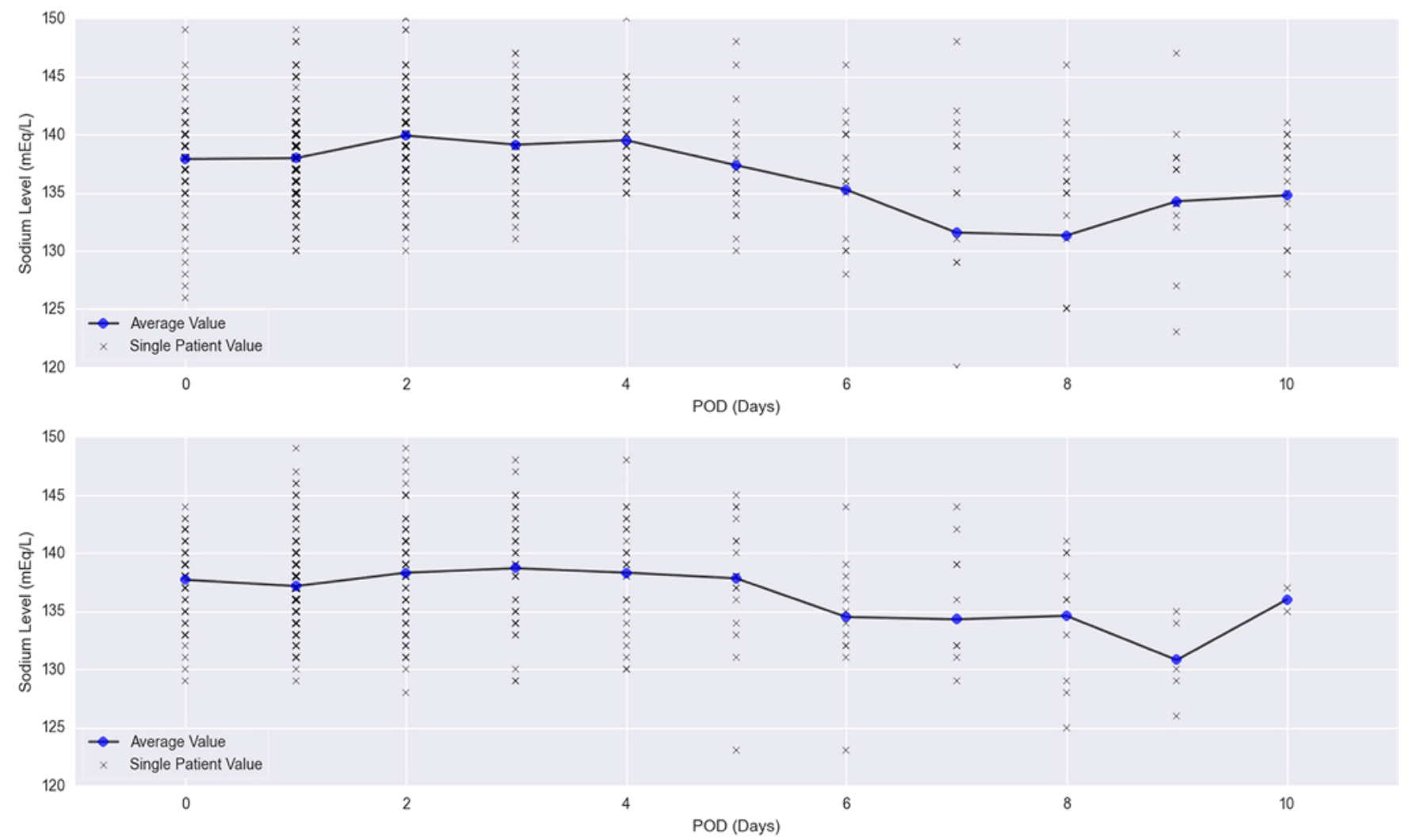

FIG. 2. Trend in serum sodium during the postoperative period of nonelderly (upper) and elderly (lower) patients undergoing TSS for NFPA resection.

Elderly patients also had a higher likelihood of developing new postoperative $\mathrm{CN}$ deficits $(1.9 \%$ vs $0.0 \%$; $\mathrm{p}=0.01$ ). Of note, only 4 patients in the elderly group had new $\mathrm{CN}$ deficits affecting CN II, III, or VII. In contrast, younger patients were more likely to develop DI (9.4\% vs $3.7 \%)$, with higher rates of transient $(5.3 \%$ vs $2.0 \%)$ and permanent (4.1\% vs $1.7 \%)$ DI than older patients $(\mathrm{p}=0.002)$. While there was no significant difference between the groups in rates of new postoperative hypopituitarism in any axis $(13.9 \%$ vs $12.0 \%$; $p=0.3)$, younger patients were more likely to develop new postoperative growth hormone/insulin-like growth factor-1 deficit $(9.5 \%$ vs $4.2 \% ; \mathrm{p}=0.03)$, without any significant differences in other hormonal axes between the groups (Table 4). In our cohort, younger patients were more likely to undergo future reoperations than older patients $(9.5 \%$ vs $3.8 \% ; \mathrm{p}=0.003)$ (Table 1$)$. Both groups also had similar rates of visual improvement after surgery $(82.7 \%$ vs $79.5 \% ; \mathrm{p}=0.5)$.

\section{Elderly Age Subgroup Comparison}

To better characterize elderly patients in our cohort, we stratified older patients into two subgroups: group 1 (age > 65 and $\leq 75$ years) and group 2 (> 75 years). Demographic and surgical outcome comparisons between the groups can be found in Table 5. Patients in group 2 had higher CCI scores (median 6.5 vs $5.0 ; p<0.001$ ). Analysis of comorbidities revealed that patients in group 2 had a higher prevalence of history of peptic ulcer disease compared with group $1(11.0 \%$ vs $2.4 \%$; $\mathrm{p}=0.01)$. Other comorbidities, demographics, and surgical outcomes were not found to be significantly different between both groups, as shown on Table 5. Regarding postoperative complications, both groups had similar rates of postoperative CSF leak requiring surgical repair $(1.7 \%$ vs $0.0 \%$; $p=0.3), 30$-day readmission $(6.2 \%$ vs $5.1 \%$; $\mathrm{p}=0.8)$, infection $(2.3 \%$ vs $2.6 \%$; $\mathrm{p}=0.999)$, postoperative hyponatremia $(24.9 \%$ vs $29.6 \%$; $\mathrm{p}=0.4)$, new postoperative $\mathrm{CN}$ deficits $(2.4 \%$ vs $1.2 \%$; $\mathrm{p}$ $=0.999)$, DI ( $4.0 \%$ vs $3.4 \% ; p=0.999)$, and new postoperative hypopituitarism $(10.3 \%$ vs $14.7 \% ; \mathrm{p}=0.4)($ Table 6$)$.

\section{Elderly CCI Score Subgroup Comparison}

To further investigate the overall health profile in elderly patients in our cohort, we stratified older patients into two subgroups by CCI score: group 1 had fewer comorbidities with CCI scores below the median of the cohort (CCI score $<6$ ), and group 2 had more comorbidities with CCI scores above the median of the cohort (CCI score $\geq 6$ ). Demographic and surgical outcome comparisons between the groups can be found in Table 5. Patients in group 2 were more likely to have diabetes mellitus (39.5\% vs $12.0 \%$; $p$ $<0.001)$, chronic obstructive pulmonary disease $(9.7 \%$ vs $2.4 \% ; \mathrm{p}=0.049)$, prior cerebrovascular accident/transient ischemic attack $(14.5 \%$ vs $3.6 \%$; $\mathrm{p}=0.01)$, chronic kidney disease $(6.5 \%$ vs $0.0 \% ; \mathrm{p}=0.02)$, congestive heart failure (6.5\% vs $0.0 \% ; p=0.02)$, previous myocardial infarction ( $13.7 \%$ vs $1.2 \% ; \mathrm{p}=0.002)$, history of peptic ulcer disease 
TABLE 5. Patient demographic information and surgical outcomes in elderly patients, stratified by age and $\mathrm{CCl}$ score

\begin{tabular}{|c|c|c|c|c|c|c|}
\hline Variable & Age $>65 \& \leq 75$ Yrs & Age $>75$ Yrs & $p$ Value & $\mathrm{CCl}<6$ & $\mathrm{CCl} \geq 6$ & $\mathrm{p}$ Value \\
\hline Total no. of patients, n (\%) & $177(60.2)$ & $117(39.8)$ & & $83(40.1)$ & $124(59.9)$ & \\
\hline Sex, $\mathrm{n}(\%)$ & & & 0.715 & & & 0.468 \\
\hline M & $109(61.6)$ & $69(59.0)$ & & $54(65.1)$ & $74(59.7)$ & \\
\hline $\mathrm{F}$ & $68(38.4)$ & $48(41.0)$ & & $29(34.9)$ & $50(40.3)$ & \\
\hline Age in yrs, median [IQR] & $70.00[67.00-72.00]$ & $79.00[77.00-83.00]$ & $<0.001$ & $69.00[67.00-74.00]$ & $76.00[71.00-81.00]$ & $<0.001$ \\
\hline LOS in days, median [IQR] & $1.00[1.00-3.00]$ & $2.00[1.00-2.00]$ & 0.509 & $1.00[1.00-3.00]$ & $2.00[1.00-3.00]$ & 0.893 \\
\hline \multicolumn{7}{|l|}{ Comorbidity } \\
\hline CCl score, median [IQR] & $5.00[5.00-6.00]$ & $6.50[6.00-7.00]$ & $<0.001$ & $5.00[4.00-5.00]$ & $6.50[6.00-7.00]$ & $<0.001$ \\
\hline \multicolumn{7}{|l|}{ Specific comorbidity, n (\%) } \\
\hline Diabetes (uncomplicated) & $34(27.2)$ & $25(30.5)$ & 0.634 & $10(12.0)$ & $49(39.5)$ & $<0.001$ \\
\hline COPD & $8(6.4)$ & $6(7.3)$ & 0.785 & $2(2.4)$ & $12(9.7)$ & 0.049 \\
\hline Peripheral vascular disease & $3(2.4)$ & $3(3.7)$ & 0.683 & $0(0.0)$ & $6(4.8)$ & 0.083 \\
\hline History of CVA/TIA & $14(11.2)$ & $7(8.5)$ & 0.641 & $3(3.6)$ & $18(14.5)$ & 0.010 \\
\hline Chronic kidney disease & $4(3.2)$ & $4(4.9)$ & 0.715 & $0(0.0)$ & $8(6.5)$ & 0.023 \\
\hline $\mathrm{CHF}$ & $2(1.6)$ & $6(7.3)$ & 0.060 & $0(0.0)$ & $8(6.5)$ & 0.023 \\
\hline Previous MI & $9(7.2)$ & $9(11.0)$ & 0.450 & $1(1.2)$ & $17(13.7)$ & 0.002 \\
\hline Mild liver disease & $5(4.0)$ & $1(1.2)$ & 0.406 & $0(0.0)$ & $6(4.8)$ & 0.083 \\
\hline Peptic ulcer disease & $3(2.4)$ & $9(11.0)$ & 0.014 & $0(0.0)$ & $12(9.7)$ & 0.002 \\
\hline Lymphoma & $2(1.6)$ & $1(1.2)$ & 0.999 & $0(0.0)$ & $3(2.4)$ & 0.276 \\
\hline Leukemia & $1(0.8)$ & $1(1.2)$ & 0.999 & $0(0.0)$ & $2(1.6)$ & 0.517 \\
\hline Connective tissue disease & $1(0.8)$ & $2(2.4)$ & 0.564 & $1(1.2)$ & $2(1.6)$ & 0.999 \\
\hline Hemiplegia & $0(0.0)$ & $1(1.2)$ & 0.396 & $0(0.0)$ & $1(0.8)$ & 0.999 \\
\hline History of metastatic tumor & $4(3.2)$ & $0(0.0)$ & 0.154 & $0(0.0)$ & $4(3.2)$ & 0.151 \\
\hline Dementia & $25(20.0)$ & $26(31.7)$ & 0.070 & $2(2.4)$ & $49(39.5)$ & $<0.001$ \\
\hline Tumor diameter in $\mathrm{cm}$, mean $\pm \mathrm{SD}$ & $2.49 \pm 1.00$ & $2.40 \pm 0.85$ & 0.436 & $2.64 \pm 1.21$ & $2.29 \pm 0.76$ & 0.012 \\
\hline CS invasion, $\mathrm{n}(\%)$ & & & 0.378 & & & 0.769 \\
\hline Yes & $55(31.6)$ & $43(36.8)$ & & $32(39.0)$ & $45(36.3)$ & \\
\hline No & $119(68.4)$ & $74(63.2)$ & & $50(61.0)$ & $79(63.7)$ & \\
\hline EOR, n (\%) & & & 0.375 & & & 0.454 \\
\hline GTR & $113(66.9)$ & $69(61.6)$ & & $52(62.7)$ & $84(68.3)$ & \\
\hline STR & $56(33.1)$ & $43(38.4)$ & & $31(37.3)$ & $39(31.7)$ & \\
\hline Drain, $n(\%)$ & & & 0.737 & & & 0.001 \\
\hline EVD & $1(0.6)$ & $0(0.0)$ & & $1(1.2)$ & $0(0.0)$ & \\
\hline Lumbar & $36(20.3)$ & $28(23.9)$ & & $18(21.7)$ & $8(6.5)$ & \\
\hline None & $140(79.1)$ & $89(76.1)$ & & $64(77.1)$ & $116(93.5)$ & \\
\hline Intraop CSF encounter, n (\%) & & & 0.999 & & & 0.241 \\
\hline Yes & $35(19.8)$ & $23(19.7)$ & & $23(27.7)$ & $25(20.2)$ & \\
\hline No & $142(80.2)$ & $94(80.3)$ & & $60(72.3)$ & $99(79.8)$ & \\
\hline Duration of op in mins, mean \pm SD & $134.7 \pm 57.1$ & $128.7 \pm 46.4$ & 0.425 & $139.1 \pm 59.8$ & $127.8 \pm 47.8$ & 0.137 \\
\hline $\mathrm{EBL}$ in $\mathrm{mL}$, mean $\pm \mathrm{SD}$ & $83.0 \pm 185.8$ & $61.0 \pm 49.4$ & 0.298 & $92.1 \pm 224.9$ & $62.3 \pm 50.4$ & 0.156 \\
\hline Adjuvant radiation, $\mathrm{n}(\%)$ & & & 0.324 & & & 0.209 \\
\hline Yes & $13(10.4)$ & $5(6.1)$ & & $10(12.0)$ & $8(6.5)$ & \\
\hline Radiosurgery & $4(3.2)$ & $3(3.7)$ & 0.999 & $2(2.4)$ & $5(4.0)$ & 0.704 \\
\hline Radiation therapy & $9(7.2)$ & $2(2.4)$ & 0.210 & $8(9.6)$ & $3(2.4)$ & 0.029 \\
\hline No & $112(89.6)$ & $77(93.9)$ & & $73(88.0)$ & $116(93.5)$ & \\
\hline Future reop, n (\%) & & & 0.759 & & & 0.480 \\
\hline Yes & $6(3.4)$ & $5(4.3)$ & & $2(2.4)$ & $6(4.8)$ & \\
\hline No & $168(96.6)$ & $111(95.7)$ & & $81(97.6)$ & $118(95.2)$ & \\
\hline
\end{tabular}

$\mathrm{CHF}=$ congestive heart failure; $\mathrm{COPD}=$ chronic obstructive pulmonary disease; $\mathrm{CVA}=$ cerebrovascular accident; $\mathrm{MI}=$ myocardial infarction; $\mathrm{TIA}=\mathrm{transient}$ ischemic attack. Boldface type indicates statistical significance. Percentages are based on the number of patients with available information for the given variable. 
TABLE 6. Postoperative complications in elderly patients, stratified by age and $\mathrm{CCl}$ score subgroups

\begin{tabular}{|c|c|c|c|c|c|c|}
\hline \multirow[b]{2}{*}{ Complication } & \multicolumn{2}{|c|}{ No. of Patients (\%) } & \multirow[b]{2}{*}{ p Value } & \multicolumn{2}{|c|}{ No. of Patients (\%) } & \multirow[b]{2}{*}{$p$ Value } \\
\hline & Age $>65 \& \leq 75$ & Age $>75$ & & $\mathrm{CCl}<6$ & $\mathrm{CCl} \geq 6$ & \\
\hline Postop CSF leak & & & 0.279 & & & 0.999 \\
\hline Yes & $3(1.7)$ & $0(0.0)$ & & $1(1.2)$ & $2(1.6)$ & \\
\hline No & $174(98.3)$ & $117(100.0)$ & & $82(98.8)$ & $122(98.4)$ & \\
\hline 30-day readmission & & & 0.802 & & & 0.526 \\
\hline Yes & $11(6.2)$ & $6(5.1)$ & & $5(6.0)$ & $5(4.0)$ & \\
\hline No & $166(93.8)$ & $111(94.9)$ & & $78(94.0)$ & $119(96.0)$ & \\
\hline Infection & & & 0.999 & & & 0.999 \\
\hline Meningitis & $3(1.7)$ & $0(0.0)$ & & $1(1.2)$ & $2(1.6)$ & \\
\hline Pneumonia & $0(0.0)$ & $1(0.9)$ & & $0(0.0)$ & $1(0.8)$ & \\
\hline Sinusitis & $1(0.6)$ & $2(1.7)$ & & $1(1.2)$ & $1(0.8)$ & \\
\hline None & $173(97.7)$ & $115(98.3)$ & & $81(97.6)$ & $120(96.8)$ & \\
\hline Postop hyponatremia & & & 0.417 & & & 0.629 \\
\hline Yes & $44(24.9)$ & $34(29.6)$ & & $23(27.7)$ & $30(24.6)$ & \\
\hline No & $133(75.1)$ & $81(70.4)$ & & $60(72.3)$ & $92(75.4)$ & \\
\hline Postop CN deficit & & & 0.999 & & & 0.651 \\
\hline Yes & $3(2.4)$ & $1(1.2)$ & & $1(1.2)$ & $3(2.4)$ & \\
\hline No & $122(97.6)$ & $81(98.8)$ & & $82(98.8)$ & $121(97.6)$ & \\
\hline DI & & & 0.999 & & & 0.006 \\
\hline Yes & $7(4.0)$ & $4(3.4)$ & & $0(0.0)$ & $10(8.1)$ & \\
\hline Transient & $4(2.3)$ & $1(0.9)$ & & $0(0.0)$ & $5(4.0)$ & \\
\hline Permanent & $3(1.7)$ & $3(2.6)$ & & $0(0.0)$ & $5(4.0)$ & \\
\hline No & $170(96.0)$ & $113(96.6)$ & & $83(100.0)$ & $114(91.9)$ & \\
\hline Postop hypopituitarism & & & 0.371 & & & 0.926 \\
\hline Yes & $12(10.3)$ & $11(14.7)$ & & $8(10.4)$ & $15(13.0)$ & \\
\hline Central hypothyroidism & $3(2.6)$ & $4(5.3)$ & 0.435 & $2(2.6)$ & $5(4.3)$ & 0.704 \\
\hline Male hypogonadism & $2(1.7)$ & $0(0.0)$ & 0.521 & $0(0.0)$ & $2(1.7)$ & 0.517 \\
\hline Female hypogonadism & $0(0.0)$ & $0(0.0)$ & - & $0(0.0)$ & $0(0.0)$ & - \\
\hline Low GH/IGF-1 & $5(4.3)$ & $3(4.0)$ & 0.999 & $3(3.9)$ & $5(4.3)$ & 0.999 \\
\hline Central hypoadrenalism & $8(6.8)$ & $9(12.0)$ & 0.300 & $7(9.1)$ & $10(8.7)$ & 0.999 \\
\hline No & $105(89.7)$ & $64(85.3)$ & & $69(89.6)$ & $100(87.0)$ & \\
\hline
\end{tabular}

Boldface type indicates statistical significance. Percentages are based on the number of patients with available information for the given variable.

( $9.7 \%$ vs $0.0 \% ; \mathrm{p}=0.002)$, and dementia $(39.5 \%$ vs $2.4 \%$; $\mathrm{p}<0.001$ ). Patients in group 1 had larger tumors (mean $2.64 \mathrm{vs} 2.29 \mathrm{~cm} ; \mathrm{p}=0.01$ ) and were more likely to require surgical drains $(22.9 \%$ vs $6.5 \%$; $\mathrm{p}=0.001)$. Although rates of adjuvant radiation therapy did not differ between the groups, in patients who required postoperative radiation treatment, group 1 was more likely to receive externalbeam radiation therapy $(9.6 \%$ vs $2.4 \% ; \mathrm{p}=0.03)$. Other comorbidities, demographics, and surgical outcomes were similar between the groups (Table 5). Patients with a CCI score $\geq 6$ were more likely to experience postoperative DI $(8.1 \%$ vs $0.0 \%$; $p=0.006)$, with similar rates of other postoperative complications (Table 6).

\section{Discussion}

The incidence of PAs in the general population is relatively high, making adenomas a common benign intracranial neoplasm. In elderly patients presenting with PA, most tumors are NFPAs. ${ }^{9}$ Because increasing life expectancy will make diagnosis of PAs in elderly patients increasingly common, evaluation of the safety of TSS for NFPAs in elderly adults is needed. Previous studies have aimed to explore outcomes after TSS for PA in this population and have reported the safety of this approach in select populations but have been limited by small sample sizes and mixed results regarding risks and complication rates. ${ }^{10,11,14-31}$

In this study, we aimed to add to the existing literature regarding TSS in the elderly. We compared nonelderly and elderly patients undergoing NFPA resection with regard to patient demographics, outcomes, and postoperative complications. To our knowledge, this study provides the largest single-institution sample size on this subject to date.

Given the increase in life expectancy and the expansion in use of MRI and CT imaging, it is presumed that the prevalence of diagnosed PAs in the elderly population 
will grow. ${ }^{21}$ Previous studies have confirmed this trend, with a steady increase in the proportion of PA cases being in elderly patients over the past 3 decades..$^{10,14,33-38}$ Our work verified this trend by revealing an overall increase in the number of patients presenting for surgical treatment of NFPA, with a parallel increase in elderly patients. However, the proportion of older patients did not significantly change over time, revealing that this increase was a reflection of a general growth in the total number of cases and not specific to their age group (Fig. 1). These nationwide trends are likely due to the aging population, increased access to diagnostic imaging, and improved surgical practice and technology allowing more elderly patients to undergo safe procedures with lower complication rates, as described here.

Interestingly, we found that younger patients were more likely to present with headaches, amenorrhea or irregular menses, galactorrhea, and decreased libido or sexual dysfunction, while older patients were more likely to be asymptomatic at the time of diagnosis (Table 2). Because providers may attribute decreased libido or sexual dysfunction in elderly patients to age-related decline, these symptoms might not be a cause for the patient to undergo MRI, which may ultimately lead to delayed presentation or diagnosis in older patients. Moreover, elderly patients may be more likely to undergo radiographic studies for various causes, such as memory impairment, mechanical falls, or workup for other malignancies and unrelated causes, and thus may have a higher rate of incidental diagnosis of NFPAs.

Regarding surgical outcomes, elderly and nonelderly patients did not differ in rates of GTR, in accordance with previous literature. ${ }^{14,20,22,39}$ LOS was also not significantly different between the groups. Previous studies have shown average LOS ranging from 3-18 days; however, in our cohort, the median LOS for elderly patients was 2 days. ${ }^{27,40}$ Similar to previous studies reporting visual symptom improvement of $71 \%-100 \%$ after surgery in these patient demographics, we found high overall rates of postoperative visual recovery and similar rates between elderly and nonelderly patients. ${ }^{14-16,19-24,31}$ Thus, as it has been previously reported by Jahangiri et al., age at surgery is not associated with postoperative normalization of vision in patients with NFPAs, but other factors such as symptom duration are more accurate predictors of postoperative visual recovery. ${ }^{41}$ Interestingly, although the number of TSSs in the elderly has increased over the past few decades, we found that younger patients were more likely to have undergone prior NFPA resection and to undergo future reoperations for recurrent or residual tumor. We also found that nonelderly adults were more likely to undergo postoperative adjuvant radiation therapy than elderly patients, especially radiosurgery. Younger patients presumably have fewer comorbidities and greater life expectancy than older patients, and thus may be more likely to undergo multiple surgeries for residual or recurrent tumors in their lifetime. In addition, given the known side effects from adjuvant therapy in addition to resection, older patients may prioritize quality of life and may choose to not undergo additional treatment for residual or recurrent disease.

TSS carries known risks of pituitary stalk perturbation leading to syndrome of inappropriate antidiuretic hormone secretion (SIADH) or DI causing hyponatremia or hypernatremia, respectively. Previous studies have documented rates of DI after TSS for pituitary pathologies as high as $18 \%$, with $2 \%-4 \%$ of patients developing permanent DI. ${ }^{42-44}$ Studies evaluating clinical outcomes in elderly patients undergoing TSS have found a similar incidence of DI in this age group, with permanent DI rates as high as 5\%.14,18,22,29,31,33 Our analysis revealed that older patients were less likely than younger patients to develop transient and permanent DI. Additionally, our subanalysis of elderly patients showed that patients with a CCI score $\geq 6$ were more likely to experience postoperative DI. In contrast, we found that older patients were more likely to develop postoperative hyponatremia than younger patients and that older patients had later peaks and troughs in postoperative serum sodium values during the postoperative course, suggesting age-related differences in stalkrelated morbidities of NFPA resection (Fig. 2). While the literature is relatively clear that postoperative DI after TSS reflects some degree of stalk injury, the mechanisms of hyponatremia after TSS are less clear but may reflect abnormal release of $\mathrm{ADH}$ or brain natriuretic peptide causing SIADH or cerebral salt wasting. ${ }^{45,46}$ It is possible that age-related structural changes in the pituitary gland, such as previously described increased interstitial and perivascular fibrosis, may confer the age-related protection against postoperative DI that we identified. ${ }^{47}$ In contrast, age-related predispositions to electrolyte perturbations due to medications that elderly patients commonly take or age-related changes in the release threshold of natriuretic factors may contribute to the age-related increase in hyponatremia we identified. ${ }^{48}$

Our study adds to the existing literature regarding the safety of TSS in elderly patients. Pereira et al. found low rates of intraoperative and postoperative complications, including $1.9 \%$ for intraoperative hypotension, $2.9 \%$ for blood transfusion, $9.6 \%$ for transient DI, $8.7 \%$ for SIADH, and $0.9 \%$ for delayed CSF leak requiring lumbar drains, with none requiring repair. ${ }^{29}$ Azab et al. also found low rates of CSF leaks, meningitis, and 30-day readmission in patients aged 60 or older. ${ }^{28}$ Additionally, Gondim et al. revealed that older patients may be more likely to experience CSF leakage, permanent DI, postoperative refractory hypertension, myocardial ischemia, and death, although rates were markedly small. ${ }^{14}$ In our cohort, we found no difference between nonelderly and elderly patients in rates of postoperative CSF leak requiring surgical repair, 30day hospital readmission, postoperative infection, and new postoperative hypopituitarism (Table 4). These findings remained true when stratifying elderly patients by age groups and CCI score to better characterize possible heterogeneity in older patients (Tables 5 and 6).

The retrospective nature of our study brings the usual limitations of a retrospective analysis. In particular, as a retrospective review, not all patients had a complete preoperative endocrine laboratory workup, which must be accounted for in regard to our findings about hypopituitarism in this study. Some inconsistencies in the frequency of outpatient sodium checks could affect our findings about hyponatremia. In addition, the data and results presented here are from surgical cases at a single institution 
with a high volume of PA patients, and, thus, the patient outcomes and postoperative complication rates reported in this paper may not be generalizable to institutions without similar volume and/or experience. Finally, although the median follow-up time did not significantly differ between the groups, it may have been too short to capture all patients requiring future reoperation for recurrence or additional long-term complications.

\section{Conclusions}

To our knowledge, we present the largest study to date examining demographics, surgical outcomes, and postoperative complications in elderly patients undergoing TSS for NFPAs. Overall, our results show that TSS for NFPA in the elderly is safe and carries positive outcomes with low complication rates. Interestingly, older patients were more likely to experience postoperative hyponatremia, whereas younger patients were more likely to develop DI. These findings, combined with our observation of higher DI in patients with more comorbidities and elderly patients experiencing a later peak and trough in serum sodium, suggest age-related differences in sodium regulation after NFPA resection. We hope that our results will help guide discussions with elderly patients regarding possible risks and outcomes.

\section{References}

1. Eltabl MA, Eladawy Y, Hanafy A, et al. Surgical outcome of endoscopic versus microscopic trans-sphenoidal approach for pituitary adenomas. Menoufia Med J. 2015;28(1):87.

2. Ezzat S, Asa SL, Couldwell WT, et al. The prevalence of pituitary adenomas: a systematic review. Cancer. 2004;101(3): 613-619.

3. Marazuela M, Astigarraga B, Vicente A, et al. Recovery of visual and endocrine function following transsphenoidal surgery of large nonfunctioning pituitary adenomas. $J$ Endocrinol Invest. 1994;17(9):703-707.

4. Gsponer J, De Tribolet N, Déruaz JP, et al. Diagnosis, treatment, and outcome of pituitary tumors and other abnormal intrasellar masses. Retrospective analysis of 353 patients. Medicine (Baltimore). 1999;78(4):236-269.

5. Lake MG, Krook LS, Cruz SV. Pituitary adenomas: an overview. Am Fam Physician. 2013;88(5):319-327.

6. Anderson GF, Hussey PS. Population aging: a comparison among industrialized countries. Health Aff (Millwood). 2000; 19(3):191-203.

7. Christensen K, Doblhammer G, Rau R, Vaupel JW. Ageing populations: the challenges ahead. Lancet. 2009;374(9696): 1196-1208.

8. United Nations Department of Economic and Social Affairs Population Division. World Population Prospects 2019: Highlights. United Nations; 2019. Accessed August 20, 2020. https://population.un.org/wpp/Publications/Files/WPP2019_ Highlights.pdf

9. Turner HE, Adams CB, Wass JA. Pituitary tumours in the elderly: a 20 year experience. Eur J Endocrinol. 1999;140(5): 383-389.

10. Hong J, Ding X, Lu Y. Clinical analysis of 103 elderly patients with pituitary adenomas: transsphenoidal surgery and follow-up. J Clin Neurosci. 2008;15(10):1091-1095.

11. Freda PU, Bruce JN. Surgery: risks of pituitary surgery in the elderly. Nat Rev Endocrinol. 2010;6(11):606-608.

12. Cooney LM, Solitaire GB. Primary intracranial tumours. Mod Geriatr. 1974;4:234-240.
13. Daly AF, Rixhon M, Adam C, et al. High prevalence of pituitary adenomas: a cross-sectional study in the province of Liege, Belgium. J Clin Endocrinol Metab. 2006;91(12): 4769-4775.

14. Gondim JA, Almeida JP, de Albuquerque LAF, et al. Endoscopic endonasal transsphenoidal surgery in elderly patients with pituitary adenomas. J Neurosurg. 2015;123(1): 31-38.

15. Kinoshita Y, Kurisu K, Arita K. Nonfunctioning pituitary adenomas in elderly patients. J Clin Neurosci. 2018;53:127-131.

16. Kurosaki M, Lüdecke DK, Flitsch J, Saeger W. Surgical treatment of clinically nonsecreting pituitary adenomas in elderly patients. Neurosurgery. 2000;47(4):843-849.

17. Asemota AO, Gallia GL. Impact of frailty on short-term outcomes in patients undergoing transsphenoidal pituitary surgery. J Neurosurg. 2019;132(2):360-370.

18. Memel Z, Chesney K, Pangal DJ, et al. Outcomes following transsphenoidal pituitary surgery in the elderly: a retrospective single-center review. Oper Neurosurg (Hagerstown). 2019;16(3):302-309.

19. Fujimoto K, Yano S, Shinojima N, et al. Endoscopic endonasal transsphenoidal surgery for patients aged over 80 years with pituitary adenomas: surgical and follow-up results. Surg Neurol Int. 2017;8(1):213.

20. Liu J, Li C, Xiao Q, et al. Comparison of pituitary adenomas in elderly and younger adults: clinical characteristics, surgical outcomes, and prognosis. J Am Geriatr Soc. 2015;63(9): 1924-1930.

21. Ferrante L, Trillò G, Ramundo E, et al. Surgical treatment of pituitary tumors in the elderly: clinical outcome and longterm follow-up. J Neurooncol. 2002;60(2):185-191.

22. Zhan R, Ma Z, Wang D, Li X. Pure endoscopic endonasal transsphenoidal approach for nonfunctioning pituitary adenomas in the elderly: surgical outcomes and complications in 158 patients. World Neurosurg. 2015;84(6):1572-1578.

23. Robenshtok E, Benbassat CA, Hirsch D, et al. Clinical course and outcome of nonfunctioning pituitary adenomas in the elderly compared with younger age groups. Endocr Pract. 2014;20(2):159-164.

24. Yunoue S, Tokimura H, Tominaga A, et al. Transsphenoidal surgical treatment of pituitary adenomas in patients aged 80 years or older. Neurosurg Rev. 2014;37(2):269-277.

25. Benbow SJ, Foy P, Jones B, et al. Pituitary tumours presenting in the elderly: management and outcome. Clin Endocrinol (Oxf). 1997;46(6):657-660.

26. Cohen DL, Bevan JS, Adams CBT. The presentation and management of pituitary tumours in the elderly. Age Ageing. 1989;18(4):247-252.

27. Wilson PJ, Omay SB, Kacker A, et al. Endonasal endoscopic pituitary surgery in the elderly. J Neurosurg. 2018;128(2): 429-436.

28. Azab MA, O'Hagan M, Abou-Al-Shaar H, et al. Safety and outcome of transsphenoidal pituitary adenoma resection in elderly patients. World Neurosurg. 2019;122:e1252-e1258.

29. Pereira EAC, Plaha P, Chari A, et al. Transsphenoidal pituitary surgery in the elderly is safe and effective. Br J Neurosurg. 2014;28(5):616-621.

30. Zhao Y, Lian W, Xing B, et al. The clinical characteristics and microsurgical therapy of pituitary adenomas in elderly patients: a retrospective study of 130 cases. J Clin Neurosci. 2017;46:13-16.

31. Marenco HA, Zymberg ST, Santos RP, Ramalho CO. Surgical treatment of non-functioning pituitary macroadenomas by the endoscopic endonasal approach in the elderly. Arq Neuropsiquiatr. 2015;73(9):764-769.

32. Charlson M, Szatrowski TP, Peterson J, Gold J. Validation of a combined comorbidity index. J Clin Epidemiol. 1994; 47(11):1245-1251.

33. Sheehan JM, Douds GL, Hill K, Farace E. Transsphenoidal 
surgery for pituitary adenoma in elderly patients. Acta Neurochir (Wien). 2008;150(6):571-574.

34. Kovacs K, Ryan N, Horvath E, et al. Pituitary adenomas in old age. J Gerontol. 1980;35(1):16-22.

35. Kurosaki M, Saeger W, Lüdecke DK. Pituitary tumors in the elderly. Pathol Res Pract. 2001;197(7):493-497.

36. Mindermann T, Wilson CB. Age-related and gender-related occurrence of pituitary adenomas. Clin Endocrinol (Oxf). 1994;41(3):359-364.

37. Pospiech J, Stolke D, Pospiech FR. Surgical treatment of pituitary adenomas in elderly patients. In: Fahlbusch R, Bock WJ, Brock M, et al., eds. Modern Neurosurgery of Meningiomas and Pituitary Adenomas. Springer Vienna; 1996:35-36.

38. Flowers A. Brain tumors in the older person. Cancer Contr. 2000;7(6):523-538.

39. Chinezu R, Fomekong F, Lasolle H, et al. Risks and benefits of endoscopic transsphenoidal surgery for nonfunctioning pituitary adenomas in patients of the ninth decade. World Neurosurg. 2017;106:315-321.

40. Grossman R, Mukherjee D, Chaichana KL, et al. Complications and death among elderly patients undergoing pituitary tumour surgery. Clin Endocrinol (Oxf). 2010;73(3):361-368.

41. Jahangiri A, Lamborn KR, Blevins L, et al. Factors associated with delay to pituitary adenoma diagnosis in patients with visual loss. J Neurosurg. 2012;116(2):283-289.

42. Nemergut EC, Zuo Z, Jane JA Jr, Laws ER Jr. Predictors of diabetes insipidus after transsphenoidal surgery: a review of 881 patients. J Neurosurg. 2005;103(3):448-454.

43. Nayak P, Montaser AS, Hu J, et al. Predictors of postoperative diabetes insipidus following endoscopic resection of pituitary adenomas. J Endocr Soc. 2018;2(9):1010-1019.

44. Little AS, Gardner PA, Fernandez-Miranda JC, et al. Pituitary gland recovery following fully endoscopic transsphenoidal surgery for nonfunctioning pituitary adenoma: results of a prospective multicenter study. J Neurosurg. Published online November 15, 2019. doi:10.3171/2019.8.JNS191012

45. Blair ET, Clemmer JS, Harkey HL, et al. Physiologic mechanisms of water and electrolyte disturbances after transsphenoidal pituitary surgery. World Neurosurg. 2017;107: 429-436.
46. Barber SM, Liebelt BD, Baskin DS. Incidence, etiology and outcomes of hyponatremia after transsphenoidal surgery: experience with 344 consecutive patients at a single tertiary center. J Clin Med. 2014;3(4):1199-1219.

47. Sano T, Kovacs KT, Scheithauer BW, Young WF Jr. Aging and the human pituitary gland. Mayo Clin Proc. 1993;68(10): 971-977.

48. Filippatos TD, Makri A, Elisaf MS, Liamis G. Hyponatremia in the elderly: challenges and solutions. Clin Interv Aging. 2017;12:1957-1965.

\section{Disclosures}

The authors report no conflict of interest concerning the materials or methods used in this study or the findings specified in this paper.

\section{Author Contributions}

Conception and design: Aghi, MP Pereira, Joshi, KM Pereira. Acquisition of data: MP Pereira, Joshi, Haddad, KM Pereira, Osorio, Donohue, Peeran, Sudhir, Jain, Beniwal. Analysis and interpretation of data: MP Pereira, Joshi, KM Pereira. Drafting the article: Aghi, MP Pereira, Oh, KM Pereira. Critically revising the article: Aghi, MP Pereira, Oh, Haddad, Gurrola, El-Sayed, Blevins, Theodosopoulos, Kunwar. Reviewed submitted version of manuscript: MP Pereira, Oh, KM Pereira, Gurrola, El-Sayed, Blevins, Theodosopoulos, Kunwar. Statistical analysis: MP Pereira, Joshi. Study supervision: Aghi, MP Pereira, Oh.

\section{Correspondence}

Manish K. Aghi: University of California, San Francisco, CA. manish.aghi@ucsf.edu. 